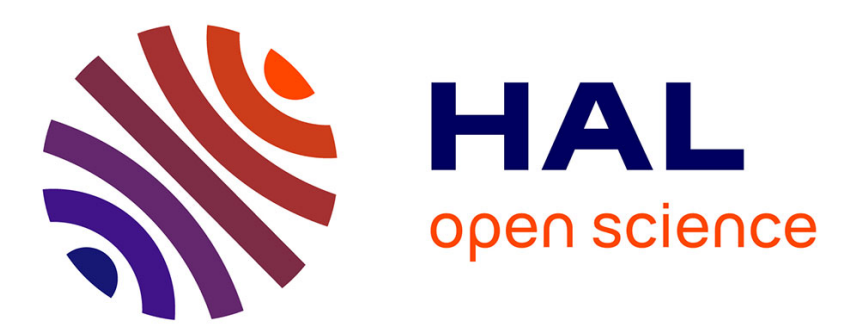

\title{
Human guided trajectory and impedance adaptation for tele-operated physical assistance
}

Guillaume Gourmelen, Benjamin Navarro, Andrea Cherubini, Gowrishankar

Ganesh

\section{- To cite this version:}

Guillaume Gourmelen, Benjamin Navarro, Andrea Cherubini, Gowrishankar Ganesh. Human guided trajectory and impedance adaptation for tele-operated physical assistance. IROS 2021 - IEEE/RSJ International Conference on Intelligent Robots and Systems, Sep 2021, Prague, Czech Republic. pp.92769282, 10.1109/IROS51168.2021.9636418 . hal-03324747

\section{HAL Id: hal-03324747 \\ https://hal.science/hal-03324747}

Submitted on 23 Aug 2021

HAL is a multi-disciplinary open access archive for the deposit and dissemination of scientific research documents, whether they are published or not. The documents may come from teaching and research institutions in France or abroad, or from public or private research centers.
L'archive ouverte pluridisciplinaire HAL, est destinée au dépôt et à la diffusion de documents scientifiques de niveau recherche, publiés ou non, émanant des établissements d'enseignement et de recherche français ou étrangers, des laboratoires publics ou privés. 


\section{Human guided trajectory and impedance adaptation for tele-operated physical assistance}

\author{
Guillaume GOURMELEN ${ }^{1} \quad$ Benjamin NAVARRO $^{1}$
}

\author{
Andrea CHERUBINI ${ }^{1}$ Gowrishankar GANESH ${ }^{1}$
}

\begin{abstract}
Human physical assistance requires the assistant to tune both his trajectory and impedance in order to assist an individual as well as be guided by him. In this study we propose a controller for teleoperated human assistance that allows the assistant to guide the assisting robot in both trajectory and impedance. We propose to use the inherent perturbations in the task, induced by the elderly or stroke patient, for impedance estimation, while a simple neuroscience based filter allows the reference estimation of the operator. We tested our impedance estimation and the controller as a whole in two experiments in which a human operator guided a robot suffering force perturbations that simulated a human patient.

Index Terms-impedance control, human guidance, teleoperation, tele-impedance, human in the loop
\end{abstract}

\section{INTRODUCTION}

In 2009, adults of age 65 or more represented $11 \%$ of the world population, and this percentage is expected to double by 2050 [1]. The percentage of elders above the age of 65 is $28 \%$ in the European Union [2], and it is expected to reach $34 \%$ in Japan by 2030 [3]. Elderly care and support, and specifically the lack of human assistants to help them, is a major concern for health-care, and in this regard robots are seen as a promising tool [4]. In this paper, we are interested in robotic elderly physical assistance, in scenarios such as lifting the person out of the bath or chair, and for assistance in feeding, which have been identified as priority tasks in elderly care [5].

A human physician or physical assistant can help a person stand up or take a cup to his/her mouth, in spite of arm tremor. In these interactions, the assistant is not (or at least, is not always) the 'leader' who imposes or forces the patient's movements. The assistant in fact acts as a 'collaborator', who aids haptically, while predicting and perceiving the motion intention [6]-[8], and constraints of the other individual. Ideally, one would like a robot assistant to be able to do the same. However, this physical collaboration requires force and impedance adaptations, and prediction of the haptic behavior, all of which are non-trivial challenges for robots. And while researchers have proposed robot controllers which mimic human impedance adaptation [9]-[11] and physical assistance

\footnotetext{
${ }^{1}$ UM-CNRS Laboratoire d'Informatique de Robotique et de Microelectronique de Montpellier (LIRMM), France

firstname.secondname@lirmm.fr

${ }^{2}$ Our demonstration video can be watched on the following link : http: //bit.do/Teleop_Impedance_Estim
}

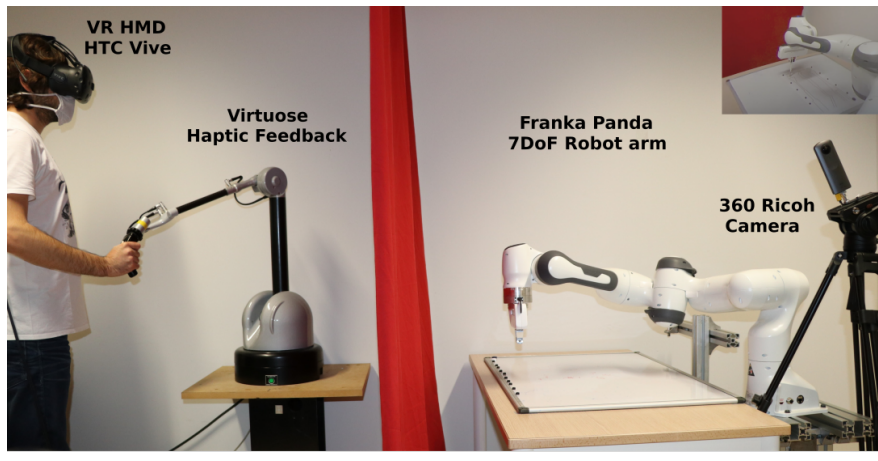

Fig. 1. Embodied teleoperation setup. Our setup consists of a Virtuose haptic device and a Franka Panda robot. The operator wears a head mounted display and operates the robot using the haptic device. He is provided with a first person visual display from a camera placed above the Franka. The controller utilized to help him guide the trajectory and impedance of the robot is explained in Sect. II. The setup can be seen in use in the demonstration video $^{2}$

[12], these controllers are reactive, and need a predefined reference, that is difficult to anticipate in an assistive scenario. It will take some time before robots will be as effective as a human assistant.

Another way of replicating the human assistant's behavior on a robot is to include him/her 'in the loop', for example via tele-operation [13]. In this case, the physical assistant drives the behavior of the assisting robot. This is the focus of our study. In regard to patient or elderly care, teleoperation cannot remove the requirement of the human assistant. Yet, it can aid one assistant help multiple individuals without going to every patient physically, hence it decreases the 'assistants over patients' ratio.

Teleoperation traditionally uses either an impedance or an admittance framework to connect the human 'leader' to the robot 'follower'; the impedance in these scenarios is either constant or adapted, but adapted relative to the environment and not the human operator [13]. Instead, for human assistance, we need a control framework that allows the transfer of impedance as well as kinematic trajectories from the human operator to the assisting follower robot. We can try to achieve this with stiff position control, but the stability of such an arrangement is not possible due to limitations of the control frequency and presence of feedback and control delays that are typical of tele-operation setups [14], [15]. An alternate method 
one may think of is to estimate the desired/reference trajectory and impedance of the human operator and implement these as an impedance controller on the robot side. While this method still suffers from performance issues due to feedback and control delays, it can be passive and more efficient in terms of the stability. This however requires one to estimate the human impedance, as well as movement reference online during task performance.

The impedance applied by a human during a movement can be estimated either by perturbing the human limb [16] or by estimating muscle activation using electromyography (EMG) or grip force. Many recent studies have utilized EMG [11], [17]-[19] or grip force [20] for human impedance estimation. Relying on muscle activation enables impedance estimation without the need for external perturbations. Besides, the changes in EMG and grip force are not only due to the limb impedance (i.e., to the stiffness and damping parameters) but are person specific, and also due to: limb motion trajectory, body posture and time (fatigue). Therefore, while EMG or grip force may still be good methods to estimate impedance in the absence of perturbations, they require user-specific calibration [21], [22]. On the other hand, in the presence of external perturbations, particularly continuous and non-repetitive ones, EMG and grip force signals include muscle reflexes, which are characterized by their own temporal and state dynamics [22] making impedance estimation non trivial.

Impedance can be estimated by adding controlled perturbations [23], but this can be detrimental for the task. Yet, in tasks like human assistance, which are themselves characterized by frequent perturbations, it is arguably better to utilize this technique - i.e., to estimate the impedance from the recorded perturbation forces and the resulting movement disturbances. Impedance measured from perturbations can be more representative - quantitatively and qualitatively - than the one estimated from muscle activation. Qualitatively, because it can enable better measures of directional impedance variations, and quantitatively because the measurement is directly at the human hand.

In this study, we propose a procedure for online (i.e., during the task) estimation of the human impedance from the perturbations. We will focus on the estimation of the stiffness and damping of the human operator, while assuming that the robot mass can be compensated for. We also propose a method, inspired by neuroscience, to estimate the reference trajectory of the human leader. Overall, our controller enables the transfer of force, trajectory and impedance, in the presence of unknown external perturbations. We test the controller in an embodied tele-assistance experiments.

The paper is organized as follows. In Sect. II, we present the tele-assistance framework, including human arm impedance parameters estimation and robot control. Next in Sect. III, we will present three experiments. In Experiment 1 we perform the validations on our human impedance estimation procedure. Then in Experiment-2, we test the impedance estimation and controller in a maze task in which human operator was required to, in some scenarios, guide the robot through a channel

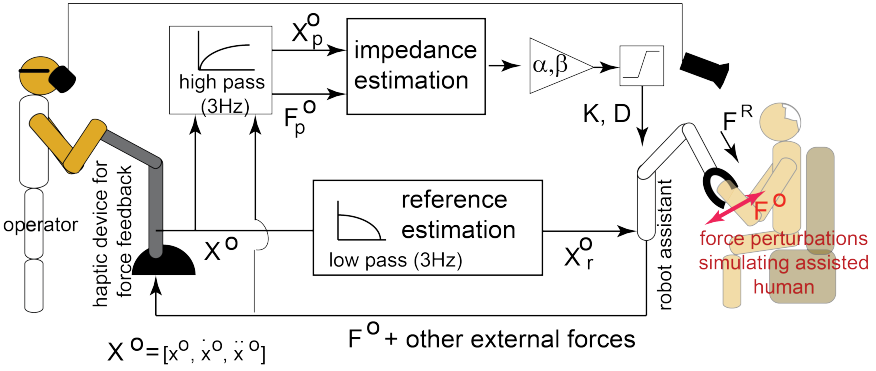

Fig. 2. Block diagram of our proposed controller and setup. The key features are the robot reference estimation and the online perturbation based impedance estimation blocks.

(a task requiring high impedance) in the presence of external disturbances (which simulated a patient) in 1-dimension, and in other scenarios, follow the directions preferred by the robot (a task requiring low impedance). In this experiment we will neglect any feedback delays, and focus on the issue of impedance and trajectory transfer assuming that popular methods of delay compensation can be utilized as such. Finally in Experiment-3 we do a stress test of the system in a scenario with 2-dimensional perturbations as well as a visual feedback delay of $500 \mathrm{~ms}$. We summarize and discuss the results in the IV section before concluding in the IV section.

\section{Methods}

We consider an operator teleoperating a fixed-base robotic manipulator using a haptic device. In such a scenario, the challenge is to properly transfer the operator's position, force, stiffness, to the robot so that the overall system feels as transparent as possible. We model the operator's Cartesian arm impedance as a mechanical mass-spring-damper system, following equation (1):

$$
\boldsymbol{F}^{O}=\boldsymbol{K} \Delta \boldsymbol{x}^{O}+\boldsymbol{D} \Delta \dot{\boldsymbol{x}}^{O}+\boldsymbol{M} \Delta \ddot{\boldsymbol{x}}^{O},
$$

where $\boldsymbol{F}^{O} \in \mathbb{R}^{6}$ is the resulting force imposed on the human operator (through our haptic device) , $\boldsymbol{K}, \boldsymbol{D}$ and $\boldsymbol{M}$ are the positive-definite $6 \times 6$ matrices of stiffness, damping and mass, and $\Delta \boldsymbol{x}^{O}=\boldsymbol{x}_{r}^{O}-\boldsymbol{x}^{O} \in \mathbb{R}^{6}$ is the error between the reference and measured positions. Here the $\mathrm{O}$ superscript stands for Operator (i.e., the human leader).

The operator's spatial state $\left[\begin{array}{lll}\boldsymbol{x}_{r}^{O} & \dot{\boldsymbol{x}}_{r}^{O} & \ddot{\boldsymbol{x}}_{r}^{O}\end{array}\right]$ is obtained using the encoder readings from the haptic device. The estimation of his/her arm's impedance parameters $[\boldsymbol{K} \boldsymbol{D} \boldsymbol{M}]$ is however not trivial. Several solutions have been proposed [18], [20], [21], [23] but all require additional and potentially intrusive hardware. To cope with this issue, we propose a method to estimate in real time the operator's arm impedance parameters as well as the operator's 'desired states' or reference using only the data available from the haptic device. We rely on known properties of the human motor system to facilitate these estimations. These parameters can then be transferred to the robot's impedance controller, to better mimic the operator's behavior and increase the system transparency. 
The whole procedure we use in our methods is described in Fig. 2. We will start by describing the reference estimation procedure in II-A, then how we extract the impedance parameters from the haptic device signals in II-B, and finish with a description of the robot controller in II-C.

\section{A. Reference Estimation}

Human movements are enabled by the simultaneous modulation of trajectory, force and impedance [24], [25]. However, the modulation of each one has properties determined by the human body sensory and mechanical constraints [26]. Here, we utilize one of these properties with regards to perturbation regulation; it has been shown that humans compensate for lower frequency perturbations by using a synchronized and opposing 'reciprocal activation' i.e., a feedforward force. On the other hand, as the perturbation frequency increases, they increase 'co-contraction' - hence impedance - to compensate for perturbations, relying completely on impedance above a certain frequency threshold [27]. This is because while human generated forces (in the absence of impacts) can contain frequencies of over $10 \mathrm{~Hz}$, the frequency of the controllable movements are much lower. While the threshold frequencies change depending on the limb in question, they decrease with the size of the limb. For the wrist, reciprocal activations fall to almost $20 \%$ of their values with a frequency of $3.5 \mathrm{~Hz}$ [27]. Thus here we hypothesized $3 \mathrm{~Hz}$ to be a suitable frequency threshold given that the perturbations disturb the whole arm in our setup.

The above observations provides us with two intuitions that help us with the reference and impedance estimation of the operator. First, because the reference trajectory is a component of the feedforward forces by the human operator, the lower frequency components of the operator states are more likely to represent his reference. And second, for impedance estimation, we should consider the high frequency components of both the operator states and operator forces, because higher frequency components are more likely to be a result of impedance and not feedforward forces or reference changes.

We therefore split the observed variable on the operator side into two components:

$$
\begin{aligned}
{\left[\begin{array}{llll}
\boldsymbol{x}_{r}^{O} & \dot{\boldsymbol{x}}_{r}^{O} & \ddot{\boldsymbol{x}}_{r}^{O}
\end{array}\right] } & =\mathrm{LPF}_{3}\left(\left[\begin{array}{lll}
\boldsymbol{x}^{O} & \dot{\boldsymbol{x}}^{O} & \ddot{\boldsymbol{x}}^{O}
\end{array}\right]\right) \\
{\left[\begin{array}{lllll}
\boldsymbol{x}^{O}{ }_{p} & \dot{\boldsymbol{x}}^{O}{ }_{p} & \ddot{\boldsymbol{x}}^{O} & { }_{p} & \boldsymbol{F}^{\boldsymbol{O}}{ }_{p}
\end{array}\right] } & =\mathrm{HPF}_{3}\left(\left[\begin{array}{llll}
\boldsymbol{x}^{O} & \dot{\boldsymbol{x}}^{O} & \ddot{\boldsymbol{x}}^{O} & \boldsymbol{F}^{\boldsymbol{O}}
\end{array}\right]\right) .
\end{aligned}
$$

In these equations, the $r$ subscript denotes the reference whereas the perturbed component of the spatial state, denoted by subscript $p$, will be used for impedance estimation, as we explain in the next section. $\mathrm{LPF}_{3}$ and $\mathrm{HPF}_{3}$ are respectively low and high pass filters with cutoff frequency $3 \mathrm{~Hz}$.

\section{B. Impedance Estimation Procedure}

The objective of the impedance estimation is to derive $\boldsymbol{K}$ and $\boldsymbol{D}$ from (1), knowning force $\boldsymbol{F}^{\boldsymbol{O}}$ generated by the haptic device, spatial state $\left[\boldsymbol{x}^{O} \dot{\boldsymbol{x}}^{O} \ddot{\boldsymbol{x}}^{O}\right]$, reference state $\left[\begin{array}{lll}\boldsymbol{x}_{r}^{O} & \dot{\boldsymbol{x}}_{r}^{O} & \ddot{\boldsymbol{x}}_{r}^{O}\end{array}\right]$ given by (2) and a priori effective cartesian mass $M$. We consider the mass of the operator to be constant, under the assumption that his/her body and arm posture do not change significantly during operation. $M$ was taken to be equal to $1 \mathrm{Kg}$ in line with human arm reach modelling studies [24]

Then from (1), we estimate $\boldsymbol{K}$ and $\boldsymbol{D}$ using least squares fit over a window of $n$ consecutive samples:

$$
\boldsymbol{A}=(\boldsymbol{F}-\boldsymbol{M} \ddot{\boldsymbol{X}}) \boldsymbol{J}_{O}^{\dagger},
$$

with:

$$
\begin{aligned}
\boldsymbol{A} & =\left[\begin{array}{ll}
\boldsymbol{K} & \boldsymbol{D}
\end{array}\right] \\
\boldsymbol{F} & =\left[\begin{array}{ll}
\boldsymbol{F} & \boldsymbol{O}_{p} \ldots \boldsymbol{F}^{\boldsymbol{O}}{ }_{p_{n}}
\end{array}\right] \\
\ddot{\boldsymbol{X}} & =\left[\begin{array}{ll}
\boldsymbol{x}^{O}{ }_{p} \ldots \boldsymbol{x}^{O}{ }_{p_{n}}
\end{array}\right] \\
\boldsymbol{J}_{O} & =\left[\begin{array}{l}
\Delta \boldsymbol{x}^{O}{ }_{p} \ldots \Delta \boldsymbol{x}^{O}{ }_{p_{n}} \\
\Delta \boldsymbol{x}^{O}{ }_{p} \ldots \Delta \boldsymbol{x}_{p_{n}}
\end{array}\right],
\end{aligned}
$$

and $\boldsymbol{J}_{O}^{\dagger}$ denotes the Moore-Penrose pseudo-inverse of $\boldsymbol{J}_{O}$.

\section{Robot control}

We consider a serial robot with $k$ degrees of freedom obeying the following dynamic model:

$$
\begin{aligned}
\boldsymbol{H}(\boldsymbol{q}) \ddot{\boldsymbol{q}}+\boldsymbol{C}(\boldsymbol{q}, \dot{\boldsymbol{q}}) \dot{\boldsymbol{q}}+\boldsymbol{g}(\boldsymbol{q}) & =\boldsymbol{\tau}^{*}+\boldsymbol{\tau}_{\mathrm{ext}} \\
\boldsymbol{H}(\boldsymbol{q}) \ddot{\boldsymbol{q}}+\boldsymbol{\tau}_{\mathrm{dyn}} & =\boldsymbol{\tau}^{*}+\boldsymbol{\tau}_{\mathrm{ext}}
\end{aligned}
$$

In these equations, $\boldsymbol{H}(\boldsymbol{q}) \in \mathbb{R}^{k \times k}$ is the inertia matrix, $\boldsymbol{C}(\boldsymbol{q}, \dot{\boldsymbol{q}}) \dot{\boldsymbol{q}} \in \mathbb{R}^{k}$ embeds Coriolis and centrifugal effects, $\boldsymbol{g}(\boldsymbol{q}) \in \mathbb{R}^{k}$ are the joint torques induced by gravity, $\boldsymbol{\tau}^{*} \in \mathbb{R}^{k}$ is the torque command and $\tau_{\text {ext }} \in \mathbb{R}^{k}$ the external torques applied to the robot. $\tau_{\text {dyn }}$ are the torques induced by Coriolis, centrifugal and gravitational forces

Since the goal of the controller is to realize Cartesian forces at the robot's end-effector, one simple way to compute $\tau^{*}$ would be to use:

$$
\boldsymbol{\tau}^{*}=\boldsymbol{J}_{R}^{\top} \boldsymbol{F}^{\boldsymbol{R}^{*}}+\boldsymbol{\tau}_{\mathrm{dyn}}
$$

where $\boldsymbol{J}_{R} \in \mathbb{R}^{6 \times k}$ is the Jacobian matrix associated with the end-effector and $\boldsymbol{F}^{\boldsymbol{R}^{*}}=\boldsymbol{F}^{\boldsymbol{R}}+\boldsymbol{F}^{\boldsymbol{O}} \in \mathbb{R}^{6}$ is the force to be realized. This is made up of two components.

The first component is our impedance controller:

$$
\boldsymbol{F}^{\boldsymbol{R}}=\boldsymbol{\alpha} \boldsymbol{K} \Delta \boldsymbol{x}^{\boldsymbol{R}}+\boldsymbol{\beta} \boldsymbol{D} \Delta \dot{\boldsymbol{x}}^{R}+\mathbf{F}_{\mathrm{I}}
$$

While the R subscript represents the robot, $\boldsymbol{F}^{\boldsymbol{R}}$ is the command force of the robot and $\boldsymbol{x}^{\boldsymbol{R}}, \dot{\boldsymbol{x}}^{R}$ are the movement and velocity of the robot relative to the reference from the operator (respectively $x_{r}^{O}$ and $\dot{x}_{r}^{O}$ ); $\boldsymbol{\alpha}$ is a scaling parameter on the human stiffness and $\boldsymbol{\beta}$ a scaling parameter on the human damping. $\mathbf{F}_{\mathbf{I}}=m^{R} \ddot{x}^{R}$ represents an approximated Cartesian inertia compensation term.

The second component, $\boldsymbol{F}^{\boldsymbol{O}}$ is a pseudo interaction force that is used in Sect. III (Experiment 2) to simulate force perturbations from an assisted patient.

However, this approach does not ensure that the robot mechanical limits are respected. To cope with this issue, we use a quadratic programming approach including the joint position, velocity and torque limits, to ensure admissibility 

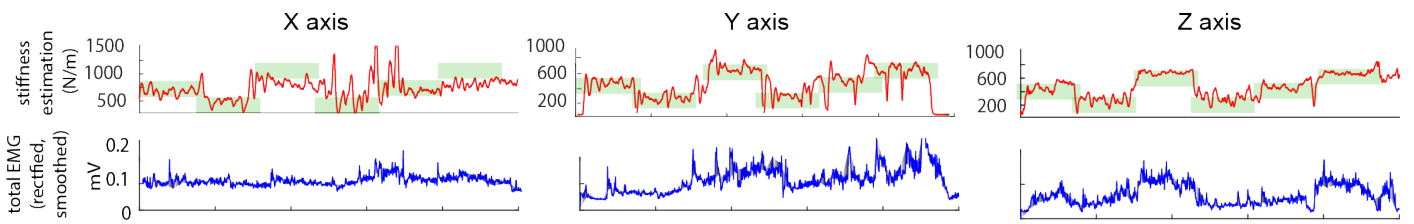

$\overline{\mathrm{J}} \overrightarrow{\mathrm{J}} \mathrm{0}, 5$
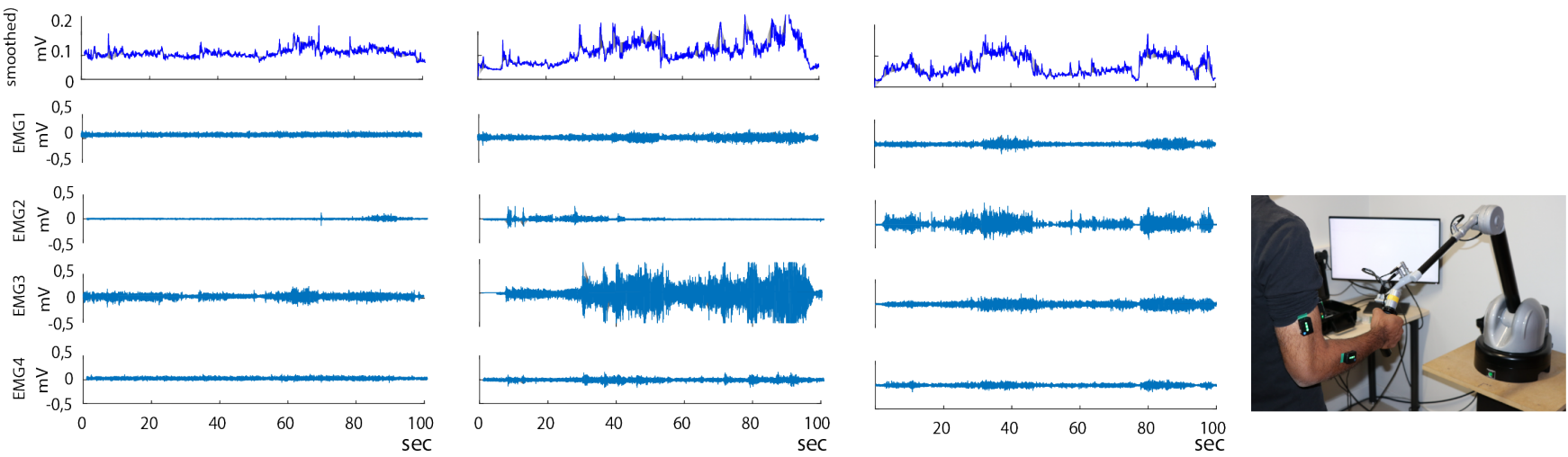

Fig. 3. Experiment 1, validation of our stiffness estimation. The participant held the Virtuose haptic device in the presence of perturbations and was provided with a feedback of the estimated stiffness by our algorithm. He was required to match his stiffness to target values displayed on the screen, represented here by cyan rectangle area in the estimation plot. We compared his stiffness changes in X (left), Y (middle), and Z (right), with the EMG recorded on four muscles and the total rectified EMG, representative of the arm impedance level. Note that the EMG just served the purpose of validating the correctness of the stiffness changes estimated by our algorithm an will not be used in our system for robot control.

of the torque control inputs. The problem is formulated as follows:

$$
\begin{array}{cl}
\underset{\boldsymbol{\tau}, \ddot{\boldsymbol{q}}}{\operatorname{minimize}} & \left\|\boldsymbol{\tau}-\boldsymbol{J}_{R}^{\top} \boldsymbol{F}^{\boldsymbol{R}^{*}}\right\|_{2}^{2} \\
\text { subject to } & \boldsymbol{\tau}=\boldsymbol{H} \ddot{\boldsymbol{q}}, \\
& \boldsymbol{\tau}_{\min }^{\prime} \leq \boldsymbol{\tau} \leq \boldsymbol{\tau}_{\max }^{\prime}, \\
& \ddot{\boldsymbol{q}}_{\min } \leq \ddot{\boldsymbol{q}} \leq \ddot{\boldsymbol{q}}_{\max } .
\end{array}
$$

In this equation: $\ddot{\boldsymbol{q}}_{\min }$ and $\ddot{\boldsymbol{q}}_{\max }$ are $k \times 1$ vectors computed to include also the joint position and velocity limits (as in [28]), $\boldsymbol{\tau}_{\min }^{\prime}$ and $\boldsymbol{\tau}_{\max }^{\prime}$ are $k \times 1$ vectors accounting for the joint torque mechanical limits $\left[-\boldsymbol{\tau}_{\max } \boldsymbol{\tau}_{\max }\right]$ with the Coriolis, centrifugal, gravity and external torques removed:

$$
\begin{aligned}
\tau_{\min }^{\prime} & =-\tau_{\max }-\tau_{\mathrm{dyn}}-\tau_{\mathrm{ext}} \\
\tau_{\max }^{\prime} & =\tau_{\max }-\tau_{\mathrm{dyn}}-\tau_{\mathrm{ext}} .
\end{aligned}
$$

Once a solution to (13) is found, the joint torque command to be sent to the robot is:

$$
\tau^{*}=\tau+\tau_{\text {dyn }} .
$$

\section{EXPERIMENTS AND RESULTS}

\section{A. Experimental Setup}

We used three experiments to verify our stiffness estimation procedure and the tele operated assistance system. The setup is depicted in Fig. 1. It consisted of a 7DOF robot arm Franka Panda [29] and a haptic feedback device Haption Virtuose 3D [30] which can feedback 3 linear forces. We utilized a HTC Vive Pro HMD [31] with a 360 degree camera to make the operator see the task from the same point of view as if the robot was his/her own arms, i.e. as if the robot was embodied [32]. To verify the correctness of the impedance estimator, we recorded Electromyography (EMG) in Experiment-1, with the Delsys Trigno wireless EMG.
During the experiments, the stiffness estimated from the perturbations was smoothed by a Butterworth low pass filter at $0.5 \mathrm{~Hz}$ (second order). On the other hand, we could not use the damping parameters calculated on the human operator and had to use the critical damping value calculated relative to the stiffness as $\boldsymbol{D}=2 \sqrt{\boldsymbol{K} \boldsymbol{M}}$ (with Mass $=1 \mathrm{Kg}$ ). We found that, probably due to the lack of an accurate mass compensation on our robot, the human calculated damping parameters were not sufficient to ensure stable performance. For security reasons, we also limited the robot stiffness values between $100 \mathrm{~N} / \mathrm{m}$ and $10000 \mathrm{~N} / \mathrm{m}$ for each Cartesian axis.

\section{B. Experiment 1: Verification of our stiffness estimation}

We started by verifying the correctness and resolution of our stiffness estimation. Unfortunately, for the ground truth, we had to rely on muscle activation, hence electromyography (EMG), which as mentioned before suffers from various limitations related to movements. To overcome them, we asked the participant to maintain a static arm posture while being disturbed by force perturbations from the haptic device. Furthermore, we chose to make the perturbations repetitive (albeit at a high frequency of $3.5 \mathrm{~Hz}$, so that the participant could not compensate via feedforward forces). The measures ensured that a constant muscle activation (hence a specific EMG level) would represent a constant impedance at the hand. In this scenario, the participants were provided with a feedback of the estimated stiffness on a computer screen (see inset of Fig. 3) while we asked them to maintain their stiffness at different target levels. We recorded EMG from four muscles in the arm (Biceps Brachii, Triceps Brachii Lateral Head, Flexi Carpi Radialis and Extensor Carpi Radialis) that were expected to contribute to the task space stiffness of the hand in our task (see plots in Fig. 3). While the EMG levels do not directly give the absolute impedance of the hand, 
the total EMG level (represented by the smooth envelope in Fig. 3) is known to correlate with the impedance, and stiffness (assuming the damping correlates with the stiffness at the hand) of the hand. We could observe different muscle pairs activating when the participants controlled their hand stiffness in the X (left column of Fig. 3), Y (middle column) and Z (right column), while the total EMG was found to co-vary with our estimated stiffness in each case.

\section{Experiment 2: Controller verification during tele- assistance}

Next, in Experiment-2, we verified how our controller performed in an assistance task and how the behavior differed when the robot impedance was kept constant. Experiment 2 had three conditions. In each condition, a 'operator' assistant guided a robot, while it helped a patient draw a line with a pen through a maze (starting with the light green semi-circles and defined by the black walls, fig 4). We did not have a real patient in the task. The patient's perturbations were simulated by force perturbations imposed on the robot, and the pen was held by the robot's two-fingers gripper. The type of perturbations and hence the impedance adaptations required by the operator were varied across the three conditions (fig $4 \mathrm{~A}, \mathrm{~B}$ and C). Parameter $\alpha$ was set to 30 in Eqn. (12).

A) One-dimensional (Y) perturbations (Fig. 4A): We started with patient perturbations only along the $\mathrm{Y}$ dimension, perpendicular to the required pen direction. The perturbations were sinusoidal, with a Frequency of $3.5 \mathrm{~Hz}$ and $10 \mathrm{~N}$ amplitude. The operator was able to control his impedance (red traces) to regulate the movement of the robot through the maze (blue traces). The $\mathrm{Y}$ displacement in time is also plotted (green trace).

B) Adaptive vs Fixed impedance (Fig. 4B): Different human assistance task require different impedances. A task requiring the human-operator to both guide (in direction) and assist (against perturbation) a patient requires higher impedances (like in our above experiment), but when the guidance is expected from the patient, better assistance is possible when the impedance of the robot is low. This variation is not possible if we use a fixed impedance on the robot. To show this, in Experiment-1B we created a scenario where the human operator assists according to guidance from the patient (again simulated by forces on the robot). Experiment $1 \mathrm{~B}$ required the human operator to close his eyes and guide the robot, while a second experimenter applied a programmed push on the robot (9 Newton force pulse in y-direction applied for $200 \mathrm{~ms}$ ) in either direction to guides the human operator away from an obstacle he would otherwise collide with. This scenario was repeated 12 times for the 2 directions $X 2$ impedance settings $(\mathrm{K}=6000 \mathrm{~N} / \mathrm{m})$ of adaptive (estimated from the operator)X 3 repetitions (see Fig. 4B). The human operator was unaware of which impedance setting and which direction of perturbation came in each trial. We calculated the absolute mean jerk in the y-direction in the trials and observed that the mean jerk in the 2 seconds after force perturbation was significantly higher for the fixed impedance condition) see bar graph in Fig. 4B).

\section{Experiment 3: Controller stress test}

Finally in Experiment-3 we made a stress test of the impedance estimation and the human- in loop controller. We introduced two changes in the task of Experiment-2A. First, the operator was subjected to 2-dimensional random perturbations and he had to adjust his impedance in two axes during the task. Second, the operator visual feedback was subjected to a delay of $500 \mathrm{~ms}$.

The results are shown in Fig. 4C. Though the operator found the task quite difficult, especially because of the visual delay, crucially we could verify that we could measure and modulate the robot impedance in two dimensions. The X-Y stiffness values during the task are plotted as stiffness ellipses which represent the estimated force for unit displacement in every direction. Note that the Eigen direction of the stiffness ellipses remain the same (while they change only in magnitude) because in this study we assume the task space $\boldsymbol{K}_{x}$ and $\boldsymbol{K}_{y}$ to be independent, and we do not consider the off diagonal terms in Eqn. (5).

\section{DISCUSSION}

In this study, we presented a human guided impedance controller during teleoperation. We designed the controller envisaging use in assistive scenarios, where a human physiotherapist or caregiver guides a follower robot to help elderly patients. These scenarios are characterized by perturbations from the individual and hence we propose to estimate the human impedance directly from the perturbations. For this purpose, we propose a methodology for online impedance estimation, and online reference estimation from the human operator.

As mentioned in the experiments, we were unable to use the damping calculated from the human operator on our robot. We found these values too low, relative to the stiffness, to ensure stable robot behavior. This comes as no surprise, given the different inertias of human arm and robot. Ideally our controller, in which the robot forces are fed to the operator and the operator movements are sent to the robot, should impose the human arm dynamics on the robot, therefore theoretically ensuring that the human damping ratios are sufficient for the robot. In practice though, this is possible only if the mass of the robot is well compensated for, which is not a trivial challenge. From human studies we know that human damping increases monotonically with stiffness, and there is still no evidence to show that humans can learn to modulate or control their damping independently from stiffness [24], [26]. Given these observations, tuning the damping separately, like we did in our experiment, seems to be a quick and sufficient solution for assistive tasks. However, further studies are required to clarify this issue.

Human interactive behaviors are enabled by simultaneous adaptations of force, trajectory and impedance. These adaptation are both predictive, to ensure stability when an interaction starts, as well as reactive, to maintain the stability during perturbations in a task. The method we propose here is specific for the measurement of reactive impedance and is arguably 


\section{Experiment 2}

A 1-dimensional (y) impedance adaptation
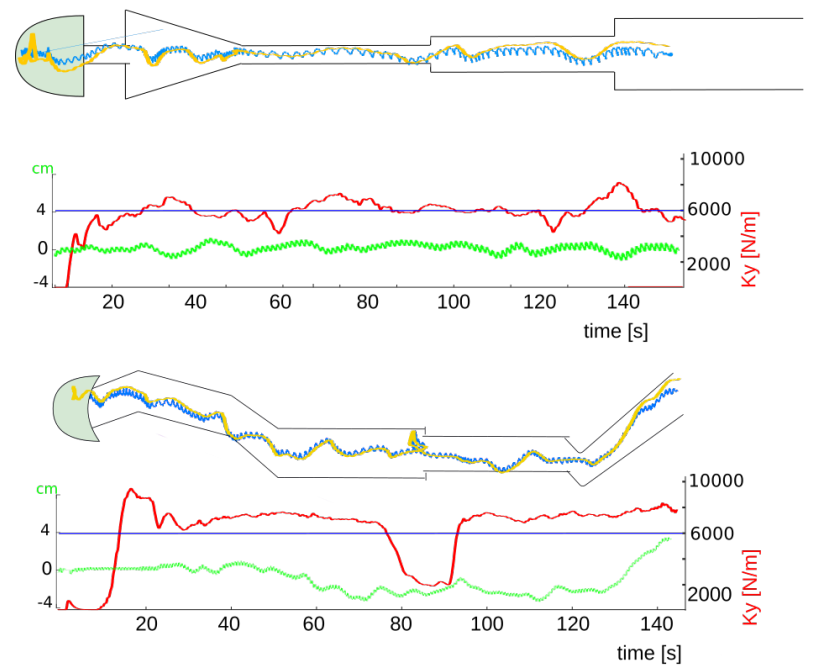

C Experiment 3 : Stress test

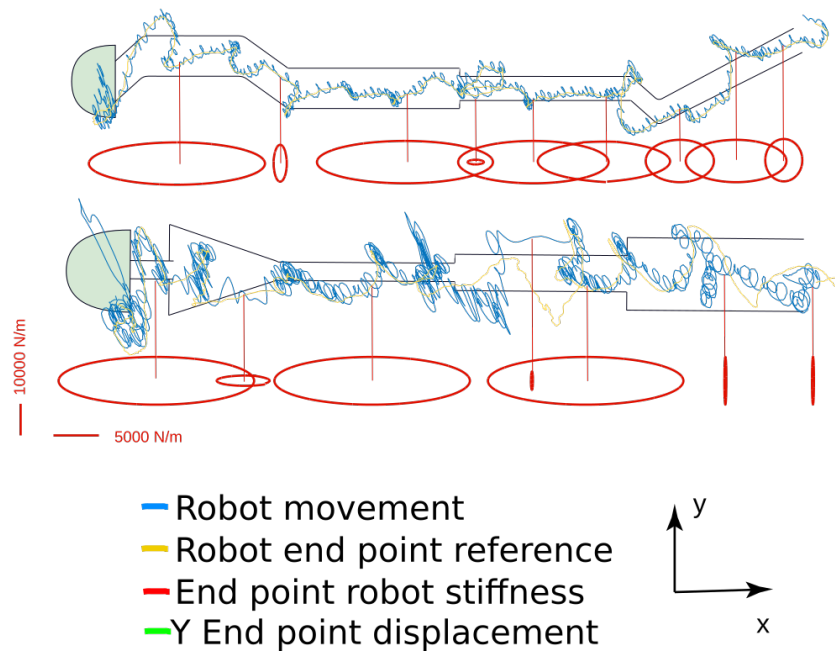

B Guidance by simulated patient

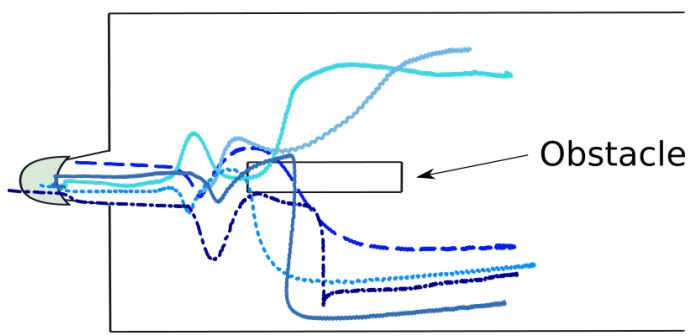

high impedance trials
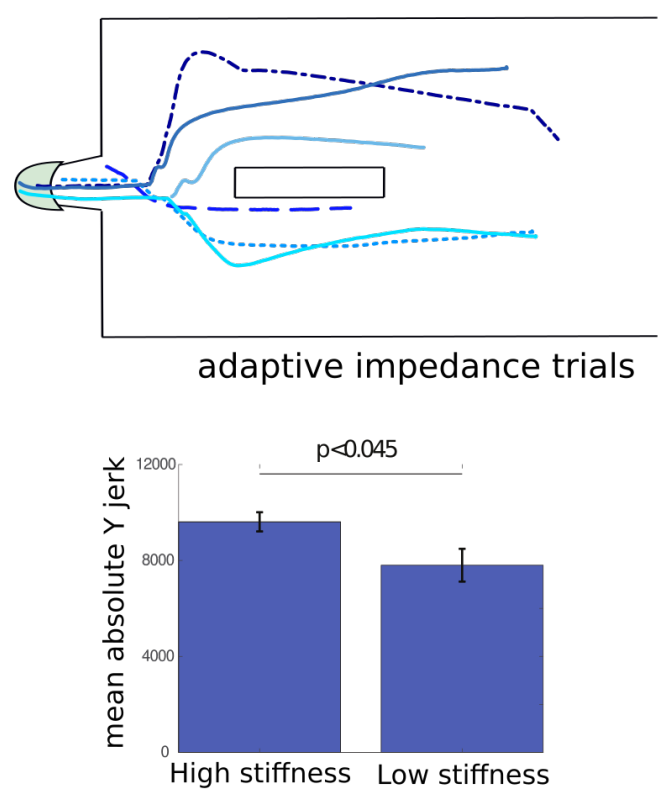

Fig. 4. Experiment 2, Assistance task. In the assistance task the operator was asked to guide the robot with a pen through a maze (starting with the light green semi-circle and defined by the black walls) while remaining inside the walls. Force perturbations on the robot simulated the disturbances from an assisted elderly individual. We performed the two experiments. Experiment 2A) The perturbations were in one dimension (Y) and the operator had to guide the robot while regulating his y-impedance to keep the robot within the walls. 2B) The operator was blind folded and asked to assist a simulated patient (simulated by the forces on our robot) while being haptically guided by the patient to avoid an obstacle. The operator worked in trials where the robot impedance was prefixed at $6000 \mathrm{~N} / \mathrm{m}$ (upper panel) or estimated from the operator (lower panel). The operator did not have prior knowledge of the condition or the direction of guidance. The average magnitude of y-jerk, observed between 0.5 seconds and 2 seconds after the guidance force was initiated, was significantly higher in case of the fixed impedance trials ( $p<0.045,2$ sample T-test). Error bars represent standard error. C) Experiment-3 served as a stress test for our system in which we introduced perturbations in two dimensions and there was a $500 \mathrm{~ms}$ delay in the visual feedback provided to the operator. The stiffness ellipses calculated in the $\mathrm{x}-\mathrm{y}$ space are shown in red and connected (with a thin red line) to the position in space where they were calculated.

better than muscle activation (EMG or grip force) based impedance estimations in the presence of perturbations (as discussed in the introduction). On the other hand, muscle activation based techniques are the only ones available for impedance estimations before the start of a movement, and in the absence of sufficient external perturbations. Robust impedance estimation in real world tasks therefore requires us to develop an integrated estimation framework in which the predictive impedance changes can be measured using muscle activation (via EMG or grip force) and the reactive changes are estimated using the perturbations, like we propose here in this study.

\section{CONCLUSION}

In conclusion, here we presented a methodology for impedance control during teleoperation with estimation and transfer of the reference and impedance from the human operator, to the robot. We provide a method of online human impedance estimation using the perturbations inherent in 
the task. This first work provided the first step towards an assistive teleoperated system for possible human assistance in the future. We are now working to update and improve the estimation of damping from the human operator, and integrating with prediction methods for the compensation of delays typical in a teleoperated system.

\section{ACKNOWLEDGMENT}

The authors want to thank Amaury Dechaux and Clementine Colomer for being participant and helping in the preparations of the experiment.

This study was supported by the JST ERATO Grant Number JPMJER1701, Japan.

\section{REFERENCES}

[1] "United nations report (2010). world aging population 2009 (publication no. st/sea/ser.a/295)." [Online]. Available: http://www. un.org/esa/population/publications/WPA2009/WPA2009-report.pdf

[2] "World bank report (2016)." [Online]. Available: http://data.worldbank. org/indicator/SP.POP.65UP.TO.ZS

[3] N. Muramatsu and H. Akiyama, "Japan: Super-aging society preparing for the future," The Gerontologist, vol. 51, no. 4, pp. 425-432, Jul. 2011. [Online]. Available: https://doi.org/10.1093/geront/gnr067

[4] J. Broekens, M. Heerink, and H. Rosendal, "Assistive social robots in elderly care: a review," Gerontechnology, vol. 8, no. 2, Apr. 2009. [Online]. Available: https://doi.org/10.4017/gt.2009.08.02.002.00

[5] "Meti 2014 revision of the four priority areas to which robot technology is to be introduced in nursing care of the elderly." [Online]. Available: http://www.meti.go.jp/english/press/2014/0203_02.html

[6] G. Ganesh, A. Takagi, R. Osu, T. Yoshioka, M. Kawato, and E. Burdet, "Two is better than one: Physical interactions improve motor performance in humans," Scientific Reports, vol. 4, no. 1, Jan. 2014. [Online]. Available: https://doi.org/10.1038/srep03824

[7] S. Kato, N. Yamanobe, G. Venture, E. Yoshida, and G. Ganesh, "The where of handovers by humans: Effect of partner characteristics, distance and visual feedback," PLOS ONE, vol. 14, no. 6, p. e0217129, Jun. 2019. [Online]. Available: https://doi.org/10.1371/journal.pone.0217129

[8] A. Takagi, F. Usai, G. Ganesh, V. Sanguineti, and E. Burdet, "Haptic communication between humans is tuned by the hard or soft mechanics of interaction," PLOS Computational Biology, vol. 14, no. 3, p. e1005971, Mar. 2018. [Online]. Available: https://doi.org/10.1371/journal.pcbi.1005971

[9] Y. Li, G. Ganesh, N. Jarrassé, S. Haddadin, A. Albu-Schaeffer, and E. Burdet, "Force, impedance, and trajectory learning for contact tooling and haptic identification," IEEE Transactions on Robotics, vol. 34, no. 5, pp. 1170-1182, 2018.

[10] C. Yang, G. Ganesh, S. Haddadin, S. Parusel, A. Albu-Schaeffer, and E. Burdet, "Human-like adaptation of force and impedance in stable and unstable interactions," IEEE Transactions on Robotics, vol. 27, no. 5, pp. 918-930, Oct. 2011. [Online]. Available: https://doi.org/10.1109/tro.2011.2158251

[11] G. Ganesh, A. Albu-Schaffer, M. Haruno, M. Kawato, and E. Burdet, "Biomimetic motor behavior for simultaneous adaptation of force, impedance and trajectory in interaction tasks," in 2010 IEEE International Conference on Robotics and Automation. IEEE, May 2010. [Online]. Available: https://doi.org/10.1109/robot.2010.5509994

[12] A. Takagi, G. Ganesh, T. Yoshioka, M. Kawato, and E. Burdet, "Physically interacting individuals estimate the partner's goal to enhance their movements," Nature Human Behaviour, vol. 1, no. 3, 2017. [Online]. Available: https://doi.org/10.1038/s41562-017-0054

[13] G. Niemeyer, C. Preusche, S. Stramigioli, and D. Lee, "Telerobotics," in Springer Handbook of Robotics. Springer International Publishing, 2016, pp. 1085-1108. [Online]. Available: https://doi.org/10.1007/ 978-3-319-32552-1_43

[14] T. Mouri, H. Kawasaki, and S. Ueki, "Bilateral tele-operated hand robot with communicational time delay," IFAC-PapersOnLine, vol. 50, no. 1, pp. 12721-12 726, 2017, 20th IFAC World Congress [Online]. Available: https://www.sciencedirect.com/science/article/pii/ S2405896317324461
[15] R. Cortesao, Jaeheung Park, and O. Khatib, "Real-time adaptive control for haptic telemanipulation with kalman active observers," IEEE Transactions on Robotics, vol. 22, no. 5, pp. 987-999, 2006.

[16] H. Gomi and M. Kawato, "Human arm stiffness and equilibriumpoint trajectory during multi-joint movement," Biological Cybernetics, vol. 76, no. 3, pp. 163-171, Apr. 1997. [Online]. Available: https://doi.org/10.1007/s004220050329

[17] L. Peternel, T. Petrič, and J. Babič, "Robotic assembly solution by human-in-the-loop teaching method based on real-time stiffness modulation," Autonomous Robots, vol. 42, no. 1, pp. 1-17, Apr. 2017. [Online]. Available: https://doi.org/10.1007/s10514-017-9635-z

[18] J. Luo, C. Yang, N. Wang, and M. Wang, "Enhanced teleoperation performance using hybrid control and virtual fixture," International Journal of Systems Science, vol. 50, no. 3, pp. 451-462, Jan. 2019. [Online]. Available: https://doi.org/10.1080/00207721.2018.1562128

[19] A. Ajoudani, N. G. Tsagarakis, and A. Bicchi, "Tele-impedance: Towards transferring human impedance regulation skills to robots," in 2012 IEEE International Conference on Robotics and Automation. IEEE, May 2012. [Online]. Available: https://doi.org/10.1109/icra.2012. 6224904

[20] D. S. Walker, R. P. Wilson, and G. Niemeyer, "User-controlled variable impedance teleoperation," in 2010 IEEE International Conference on Robotics and Automation. IEEE, May 2010. [Online]. Available: https://doi.org/10.1109/robot.2010.5509811

[21] A. Ajoudani, Transferring Human Impedance Regulation Skills to Robots. Springer International Publishing, 2016. [Online]. Available: https://doi.org/10.1007/978-3-319-24205-7

[22] L. M. Doornebosch, D. A. Abbink, and L. Peternel, "Analysis of coupling effect in human-commanded stiffness during bilateral tele-impedance," IEEE Transactions on Robotics, pp. 1-16, 2021. [Online]. Available: https://doi.org/10.1109/tro.2020.3047064

[23] M. D. Hill and G. Niemeyer, "Real-time estimation of human impedance for haptic interfaces," in World Haptics 2009 - Third Joint EuroHaptics conference and Symposium on Haptic Interfaces for Virtual Environment and Teleoperator Systems. IEEE, 2009. [Online]. Available: https://doi.org/10.1109/whc.2009.4810893

[24] D. M. Wolpert, J. Diedrichsen, and J. R. Flanagan, "Principles of sensorimotor learning," Nature Reviews Neuroscience, vol. 12, no. 12, pp. 739-751, Oct. 2011. [Online]. Available: https://doi.org/10.1038/ nrn3112

[25] G. Ganesh and E. Burdet, "Motor planning explains human behaviour in tasks with multiple solutions," Robotics and Autonomous Systems, vol. 61, no. 4, pp. 362-368, Apr. 2013. [Online]. Available: https://doi.org/10.1016/j.robot.2012.09.024

[26] T. E. M. Etienne Burdet, David W. Franklin, Human Robotics. MIT Press, 2013, vol. 978-0-262-01953-8. [Online]. Available: https://mitpress.mit.edu/books/human-robotics

[27] M. E. B. . G. Ganesh, A. Melendez-Calderon, "Transition between reciprocal activation and co-contraction during wrist posture control,' 2020. [Online]. Available: https://hal.archives-ouvertes.fr/hal-02974058

[28] K. Bouyarmane, J. Vaillant, K. Chappellet, and A. Kheddar, "Multirobot and task-space force control with quadratic programming," Mar. 2017, working paper or preprint. [Online]. Available: https: //hal.archives-ouvertes.fr/hal-01495662

[29] "Franka panda emika 7dof torque controlled robot arm." [Online]. Available: https://www.franka.de/technology

[30] "Haption virtuose 3d, haptic feedback device." [Online]. Available: https://www.haption.com/fr/products-fr/virtuose-3d-fr.html

[31] "Vr head mounted display htc vive." [Online]. Available: https: //www.vive.com/fr/product/vive-pro/

[32] A. Toet, I. A. Kuling, B. N. Krom, and J. B. F. van Erp, "Toward enhanced teleoperation through embodiment," Frontiers in Robotics and AI, vol. 7, Feb. 2020. [Online]. Available: https://doi.org/10.3389/frobt.2020.00014 